\title{
Speckle Interferometry of Metal-Poor Stars in the Solar Neighborhood. I
}

\author{
D. A. Rastegaev, Yu. Yu. Balega, E. V. Malogolovets \\ Special Astrophysical Observatory, RAS, Nizhnii Arkhyz, Karachai-Cherkessian Republic, 357147 Russia \\ received: June 15, 2007/revised: June 28, 2007
}

\begin{abstract}
We report the results of speckle-interferometric observations of 109 high proper-motion metal-poor stars made with the 6-m telescope of the Special Astrophysical Observatory of the Russian Academy of Sciences. We resolve eight objects — G102-20, G191-55, BD+19 1185A, G89-14, G87-45, G87-47, G111-38, and G114-25 - into individual components and we are the first to astrometrically resolve seven of these stars. New resolved systems included two triple (G111-38, G87-47) and one quadruple (G89-14) star. The ratio of single-to-binary-totriple-to-quadruple systems among the stars of our sample is equal to 71:28:6:1.
\end{abstract}

\section{INTRODUCTION}

Stars of the halo and thick disk of our Galaxy are old metal-poor objects with large spatial velocities (Norris, 1986, Majewski, 1993). The studies of these stars can be used to impose constraints on the physical conditions during the early stages of the formation of our Galaxy. Binary and multiple stars are the best candidate objects to be used for studying the process of star formation at the time of formation of our Galaxy, because they bear more information about this process compared to single stars. This information is coded both in the orbital parameters (eccentricity, semi-major axis) and physical parameters of the components (component luminosities and the mass function).

The authors of early papers dedicated to the study of the multiplicity of the old population of the Galaxy (Abt \& Levi, 1969 Abt \& Willmarth, 1987) concluded that the fraction of binary and multiple systems among these objects is very low compared to the corresponding fractions for younger stars of the Galactic disk, which are richer in heavy elements. However, the picture has changed in the last two decades. In series of papers opened by (Carney \& Latham, 1987) is shown that the binary-to-single star ratio for halo and thick-disk stars is comparable to the corresponding ratio for the overwhelming majority of stars in the solar neighborhood. Such studies are based on the analysis of stellar spectra (Goldberg et al., 2002, Latham et al., 2002) using the data on visual binaries and common proper motion pairs (Allen et al., 2000, Zapatero Osorio \& Martin, 2004).

Send offprint requests to: D. A. Rastegaev, e-mail: leda@sao.ru
We still have insufficient data about the multiple old stars in the solar neighborhood with orbital semimajor axes in the interval from $\sim 1$ to $\sim 100 \mathrm{AU}$ observable with adaptive optics and speckle-interferometric facilities. We point out the paper by Zinnecker et al. (Zinnecker et al., 2004) who report the results of observations of population II stars using the techniques of speckle interferometry, adaptive optics, and direct imaging. To expand the database on such objects and determine the properties of the components of multiple systems, we began speckle-interferometric observations of metal-poor objects with large proper motions located in the close vicinity of the Sun. In this paper we report the results of observations of 109 halo and thick-disk stars made during the period from April through December, 2006.

The paper has the following layout: Section 2 describes the sample of stars studied; Section 3 analyzes the methods of observations and reduction of the data obtained; Sections 4 and 5 list the results of observations and additional information about the resolved stars, respectively; Section 6 discusses the multplicity of the stars studied, and the last section gives the conclusions.

\section{THE SAMPLE}

We selected our program stars from the CLLA catalog (Carney et al., 1994). This catalog is actually a sample of A- to early K-type dwarfs from the Lowell Proper Motion Catalog (Giclas et al., 1971, Giclas et al., 1978), which contains mostly Northern-Hemisphere stars brighter than 16 magnitude with proper motions exceeding 0.26 " /year.

We selected a total of 223 stars from the CLLA catalog based on the following three criteria:

$$
\text { 1. }[\mathrm{m} / \mathrm{H}]<-1 \text {; }
$$




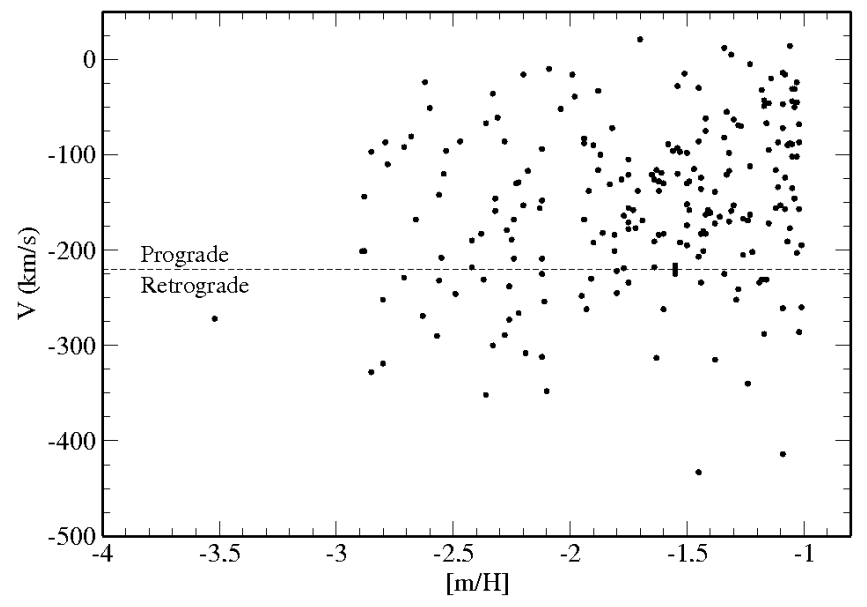

Fig. 1. Distribution of the stars of the sample by metallicity $[\mathrm{m} / \mathrm{H}]$ and $\mathrm{V}$ component of spatial velocity. The dashed line separates stars moving in prograde and retrograde orbits.

\section{2. $\delta>-10^{\circ}$}

3. $\mathrm{m}_{\mathrm{V}}<12$.

We use the data of the CLLA catalog to make several figures illustrating some of the main characteristics of our sample. Figure 1 shows the distribution of our selected stars on the "metallicity - V-component of the spatial velocity" plane, which demonstrates that our stars belong to different components of the Galaxy. The left-hand part of the diagram is occupied by halo objects - metal-poor stars with high velocity dispersion. The upper right corner is populated by stars belonging to the metal-weak tail of the thick disk. About $20 \%$ of all stars (45 objects) move in retrograde orbits. Figure 2 shows the distribution of the heliocentric distances of the stars of our sample. We used the photometric distances from the CLLA catalog. Arifyanto et al. (Arifyanto et al., 2005) compared the photometric parallaxes with the corresponding trigonometric parallaxes measured by Hipparcos (Perryman, 1997) for stars of the catalog considered and showed that there is a small discrepancy between the heliocentric distances determined using different methods. However, we did not correct the photometric distances in any way. Fig. 3 shows the distribution of the metallicities of the stars studied. The $[\mathrm{m} / \mathrm{H}]<-3$ metallicity interval is represented by only one star, G64-12, with $[\mathrm{m} / \mathrm{H}]=-3.52$. Half of the stars studied have metallicities in the interval $[\mathrm{m} / \mathrm{H}]=$ $[-1.58 ;-1)$. As is evident from the distribution of stellar temperatures (Fig. 44), these are F-, G-, and K-type stars.

\section{OBSERVATIONS AND DATA REDUCTION}

We performed speckle-interferometry of 109 stars of the sample with the 6-m telescope of the Special Astrophysical Observatory of the Russian Academy of Sciences (SAO RAS): in April (one star), May (five objects), June (six objects), and December, 2006 (97 objects). Before December 2006 observations were made using a facility described by Maksimov et al. (Maksimov et al., 2003). Its detector con-

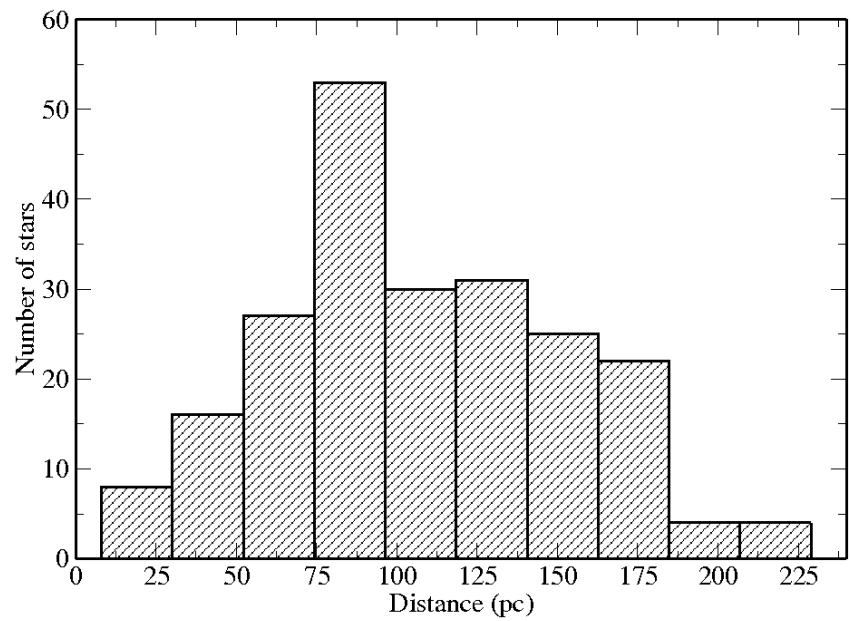

Fig. 2. Distribution of heliocentric distances of the stars of the sample (we adopt the distances from the CLLA catalog).

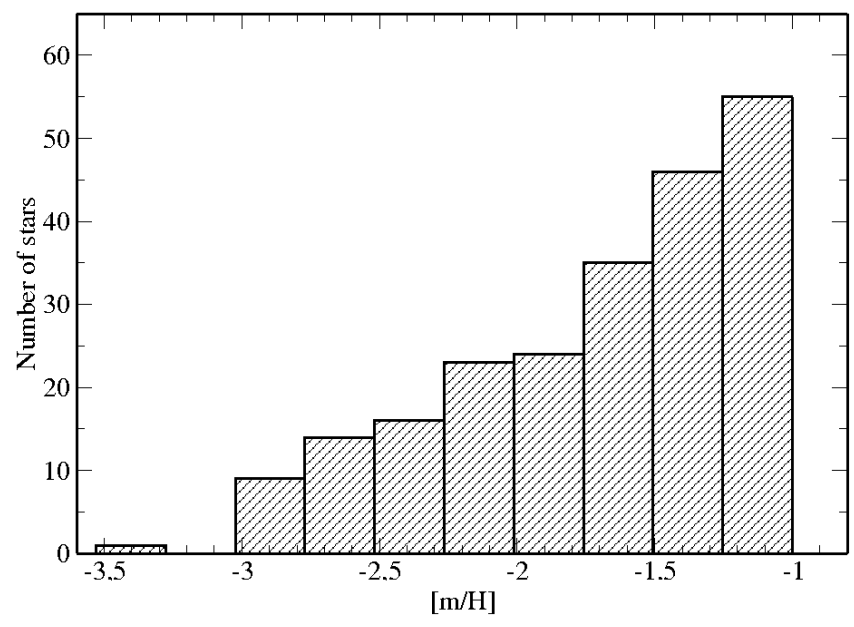

Fig. 3. Distribution of the metallicities of the stars of the sample (we adopt the metallicities from the CLLA cata$\log )$.

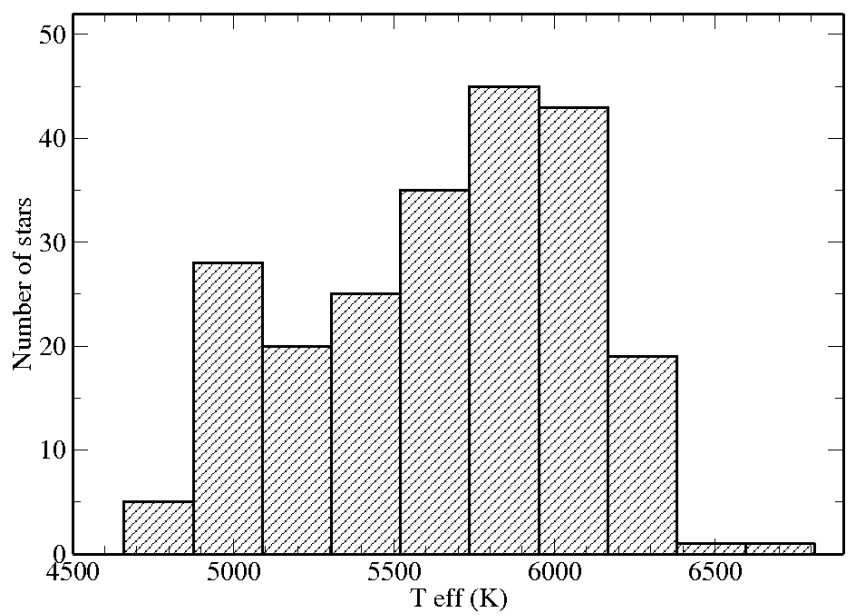

Fig. 4. Distribution of the temperatures of the stars of the sample (we adopt the temperatures from the CLLA (atalog). 
sists of a fast $1280 \times 1024$ Sony ICX085 CCD combined with a three-stage image intensifier with electrostatic focusing. In December we used a new facility based on an EMCCD (a CCD with internal electron gain) with higher quantum efficiency and better linearity. Both facilities are capable of detecting objects with component magnitude differences up to $4^{m}$.

We recorded speckle interferograms in filters with the parameters of $545 / 30 \mathrm{~nm}$ (the first and the second numbers give the central wavelength and half bandwidth of the filter, respectively), 550/20,800/110, and 800/100 nm with exposures ranging from five to 20 milliseconds. In December 2006 we obtained 500 short-exposure images for each of most of the objects. For each of the remaining objects 2000 exposures are accumulated.

A description of the technique used to determine the relative positions and component magnitude differences from the power spectra of speckle interferograms averaged over the set can be found in Balega et al., 2002. The diffraction limit of the resolution is equal to $0.022^{\prime \prime}$ in the $545 / 30$ and $550 / 20 \mathrm{~nm}$ filters and $0.033^{\prime \prime}$ in the $800 / 110$ and 800/100 filters. The measured position angles and angular separations $\rho$ are accurate to $0.3-2.8^{\circ}$ and 1 to 5 mas, respectively. The errors of measured $\theta$ and $\rho$ depend on a number of parameters: component separation, magnitude difference, and seeing $\beta$. The accuracy of the component magnitude difference determined from the power spectrum is also a function of the same parameters. It usually varies from $0.05^{m}$ to $0.2^{m}$ for $m_{V}=8-10$ objects.

\section{RESULTS OF OBSERVATIONS}

The main results of this work are listed in Tables 1 and 4

We resolved into components the following eight objects: G102-20, G191-55, BD+19 ${ }^{\circ} 1185 \mathrm{~A}$, G89-14, G8745, G87-47, G111-38, and G114-25. We astrometrically resolved seven among these eight objects for the first time and found new components in five objects: G191-55, G8914, G87-47, G111-38, and G114-25. We were the first to astrometrically resolve the well-known spectroscopic binary G102-20 (Latham et al., 2002). We are also the first to establish the astrometric binarity of the G87-45 system whose spectrum exhibits signatures of three components (Latham et al., 2002). The astrometric binarity of the resolved object $\mathrm{BD}+19^{\circ} 1185 \mathrm{~A}$ was earlier discovered by Hipparcos (Perryman, 1997).

Table 2 gives some data on resolved systems. The last column of this table summarizes the results of all available astrometric and spectroscopic observations of the objects studied including the speckle-interferometric observations performed with the 6 -m telescope of SAO RAS.

\section{ADDITIONAL DATA ON RESOLVED STARS}

In this section we summarize additional data on the resolved stars. For some objects we list two distances determined from photometric (Carney et al., 1994) and trigonometric (Perryman, 1997) parallaxes. It is evident that the distance determined using the former method is underestimated, because it does not take into account the luminosity of the additional component. On the other hand, the additional component also introduces certain error in the measured trigonometric parallaxes, especially for short-period systems.

G102-20 $\left(05^{h} 40^{m} 09^{\mathrm{s}} .7+12^{\circ} 10^{\prime} 41^{\prime \prime}\right.$; HIP 26676$)$. This known SB1-type spectroscopic binary with a 26-year period (Latham et al., 2002) and a heliocentric distance of $\approx 70$ pc (Perryman, 1997) was resolved speckleinterferometrically for the first time.

G191-55 $\left(05^{h} 57^{m} 28^{\mathrm{s}} .6+58^{\circ} 40^{\prime} 49^{\prime \prime} ; \quad \mathrm{BD}+58^{\circ} \quad 876 ;\right.$ TYC 3762-1904-1). It is an F8-type binary (SIMBAD) located at a heliocentric distance of $\approx 93 \mathrm{pc}$ (Carney et al., 1994).

$\mathbf{B D}+\mathbf{1 9}^{\circ} \mathbf{1 1 8 5} \mathbf{A} \quad\left(06^{h} 03^{m} 14.9 \quad+19^{\circ} 21^{\prime} 39^{\prime \prime} ;\right.$ HIP 28671). It is a G0V-type object (SIMBAD). The binary nature of this star with $\rho=223$ mas was discovered by Hipparcos. Its heliocentric distance is estimated at $d \approx 42$ (Carney et al., 1994) and $d \approx 66 \mathrm{pc}$ (Perryman, 1997). This is a triple system given the presence of a distant companion $\left(\rho \approx 7^{\prime \prime}\right) \mathrm{BD}+19^{\circ} 1185 \mathrm{~B}$.

G89-14 $\left(07^{h} 22^{m} 31^{\mathrm{s}} .5 \quad+08^{\circ} 49^{\prime} 13^{\prime \prime} ; \quad\right.$ HIP 35756 ; WDS $07224+0854$ ) is a new quadruple system. We resolved it as a pair with a separation of $0.99^{\prime \prime}$. Allen et al. Allen et al., 2000 provide evidence suggesting that the system may contain a physically bound component at a distance of $34^{\prime \prime}$. At the same time, G89-14 is an SB1-type spectroscopic binary with a period of 190 days (Latham et al., 2002). The star is at a heliocentric distance of $\approx 94 \mathrm{pc}$ (Perryman, 1997).

G87-45 $\left(07^{h} 32^{m} 58^{\mathrm{s}} .7+31^{\circ} 07^{\prime} 00^{\prime \prime}\right.$; TYC 2453-763-1). It is a G2-type star (SIMBAD) known as an SB2type spectroscopic binary with a period of 51 days (Latham et al., 1988). The spectrum of this star exhibits signs of a third component (Latham et al., 2002), which we must have resolved. The distance to the star is $d \approx$ 123 pc (Carney et al., 1994).

G87-47 $\left(07^{h} 35^{m} 34^{\mathrm{s}} 1+35^{\circ} 57^{\prime} 11^{\prime \prime}\right.$; HIP 36936). A new triple system whose distance is estimated at $d \approx 62$ (Carney et al., 1994) and $d \approx 100$ pc (Perryman, 1997). The system is known as an SB1-type spectroscopic binary with a period of 13 days. The Hipparcos catalog lists it as an object with stochastic astrometric solution. We found a third component. For this object we could determine the position of the secondary only with an uncertainty of $\pm 180^{\circ}$.

G111-38 $\left(07^{h} 49^{m} 32^{\mathrm{s}} 0 \quad+41^{\circ} 28^{\prime} 08^{\prime \prime} ;\right.$ HIP 38195 ; WDS $07495+4128)$. A new triple system. Hipparcos resolved it as a binary with $\rho=2.154^{\prime \prime}$. We resolved one of the components. The distance to the system is estimated at $d \approx 50 \mathrm{pc}$ (Carney et al., 1994) or $d \approx 200 \mathrm{pc}$ 
Table 1. Speckle-interferometric measurements of resolved objects

\begin{tabular}{l|c|c|c|c}
\hline $\begin{array}{l}\text { Name of } \\
\text { the system/ } \\
\text { subsystem }\end{array}$ & $\rho\left(^{\prime \prime}\right)$ & $\Theta\left(^{\circ}\right)$ & $\triangle m$ & Filter \\
\hline G102-20 & $0.120 \pm 0.006$ & $308.0 \pm 2.8$ & $3.24 \pm 0.11$ & $550 / 20$ \\
G191-55 & $0.814 \pm 0.002$ & $125.1 \pm 0.3$ & $2.00 \pm 0.01$ & $800 / 100$ \\
BD+19 1185A & $0.115 \pm 0.001$ & $183.6 \pm 0.7$ & $1.77 \pm 0.02$ & $550 / 20$ \\
G89-14 & $0.989 \pm 0.005$ & $0.8 \pm 0.4$ & $4.14 \pm 0.06$ & $800 / 100$ \\
G87-45 & $0.285 \pm 0.002$ & $271.3 \pm 0.5$ & $2.01 \pm 0.04$ & $550 / 20$ \\
G87-45 & $0.285 \pm 0.002$ & $270.7 \pm 0.4$ & $1.76 \pm 0.02$ & $800 / 100$ \\
G87-47 & $0.078 \pm 0.003$ & $54.0^{*} \pm 2.1$ & $1.74 \pm 0.03$ & $800 / 100$ \\
G111-38AB & $0.084 \pm 0.001$ & $7.9 \pm 0.7$ & $0.78 \pm 0.01$ & $550 / 20$ \\
G111-38AB & $0.084 \pm 0.001$ & $7.8 \pm 1.3$ & $0.75 \pm 0.01$ & $800 / 100$ \\
G111-38AC & $2.133 \pm 0.005$ & $200.0 \pm 0.3$ & $1.34 \pm 0.01$ & $550 / 20$ \\
G111-38AC & $2.133 \pm 0.005$ & $200.0 \pm 0.3$ & $1.10 \pm 0.01$ & $800 / 100$ \\
G111-38BC & $2.216 \pm 0.005$ & $199.5 \pm 0.3$ & $0.57 \pm 0.02$ & $550 / 20$ \\
G111-38BC & $2.216 \pm 0.005$ & $199.5 \pm 0.3$ & $0.36 \pm 0.03$ & $800 / 100$ \\
G114-25 & $0.781 \pm 0.005$ & $323.7 \pm 0.5$ & $3.83 \pm 0.16$ & $800 / 100$ \\
\hline
\end{tabular}

* The position of the secondary componentis known with an uncertainty of $\pm 180^{\circ}$.

Table 2. Additional data on resolved stars

\begin{tabular}{l|c|c|c|c}
\hline $\begin{array}{l}\text { Name of the } \\
\text { system/ } \\
\text { subsystem }\end{array}$ & $\begin{array}{c}\text { Coordinates } \\
(2000.0)\end{array}$ & $\mathrm{m}_{V}$ & {$[\mathrm{~m} / \mathrm{H}]^{*}$} & $\begin{array}{c}\text { Total multiplicity } \\
\text { of the system }\end{array}$ \\
\hline G102-20 & $05^{h} 40^{m} 09^{\mathrm{s}} .7+12^{\circ} 10^{\prime} 41^{\prime \prime}$ & 10.22 & -1.17 & 2 \\
$\mathrm{G} 191-55$ & $05^{h} 57^{m} 28^{\mathrm{s}} .6+58^{\circ} 40^{\prime} 49^{\prime \prime}$ & 10.47 & -1.94 & 2 \\
$\mathrm{BD}+19^{\circ} 1185 \mathrm{~A}$ & $06^{h} 03^{m} 14^{\mathrm{s}} .9+19^{\circ} 21^{\prime} 39^{\prime \prime}$ & 9.32 & -1.47 & 3 \\
$\mathrm{G} 89-14$ & $07^{h} 22^{m} 31^{\mathrm{s}} .5+08^{\circ} 49^{\prime} 13^{\prime \prime}$ & 10.40 & -1.90 & 4 \\
$\mathrm{G} 87-45$ & $07^{h} 32^{m} 58^{\mathrm{s}} .7+31^{\circ} 07^{\prime} 00^{\prime \prime}$ & 11.44 & -1.49 & 3 \\
$\mathrm{G} 87-47$ & $07^{h} 35^{m} 34^{\mathrm{s}} .1+35^{\circ} 57^{\prime} 11^{\prime \prime}$ & 10.34 & -1.34 & 3 \\
$\mathrm{G} 111-38$ & $07^{h} 49^{m} 32^{\mathrm{s}} .0+41^{\circ} 28^{\prime} 08^{\prime \prime}$ & 8.7 & -1.04 & 3 \\
$\mathrm{G} 114-25$ & $08^{h} 59^{m} 03^{\mathrm{s}} .4-06^{\circ} 23^{\prime} 46^{\prime \prime}$ & 11.92 & -2.28 & 2 \\
\hline
\end{tabular}

${ }^{*}$ Metallicities adopted from the CLLA catalog (Carney et al., 1994).

(Perryman, 1997). The spectral type of the system is G5 (SIMBAD).

G114-25 $\left(08^{h} 59^{m} 03^{\mathrm{s}} 4-06^{\circ} 23^{\prime} 46^{\prime \prime}\right.$; HIP 44111). A new binary of spectral type F7 (SIMBAD). Its heliocentric distance is $d \approx 131$ pc (Carney et al., 1994).

\section{MULTIPLICITY OF STARS}

\subsection{Distant Components}

For 109 of the objects considered we used additional data on the spectroscopic multiplicity of these stars (Goldberg et al., 2002 Latham et al., 2002) and the data on distant components from the WDS (Mason et al., 2001) catalog. Whereas spectroscopic and interferometric components appear to be undoubtedly physically bound, one must treat wide visual companions more carefully. We found a total of 43 WDS components (in some cases several components for one object), most of which we rejected as accidental optical projections. Table 3 lists the data on the all found wide components. The first column gives the names of the stars studied and the second column, all the WDS components found. For the components found to be physically bound to the corresponding stars columns 3 and 4 give the component separation and magnitude difference, respectively. We adopt the latter from the WDS catalog and they may differ slightly from the quantities given in the corresponding references. The '+' and '-' signs in column 5 (Status) mark the components, which we consider to be possibly physically bound to the main star and physically unbound optical pairs, respectively. A question mark in this column indicates that we are not sure about our decision. The last column gives the references to the papers, which contain data on the corresponding pair and confirm or disprove its physical relationship. In all cases these are two papers (Allen et al., 2000; Zapatero Osorio \& Martin, 2004) dedicated to wide pairs of population II stars and the Hipparcos catalog (Perryman, 1997). Additional $\star$ symbol in this column indicates that our observations confirm the presence of the given component. If no references are given, it means that we made our own decision about the physical association based on the data provided by the WDS catalog. To this end, we analyzed the component magnitude difference and the change of the component separations over the time periods covered by observations. 
As a result, we left only 12 objects among the initial 43 WDS components and used them to compute the ratios of the number of systems of different degree of multiplicity.

\subsection{Ratio of the Systems of Different Degree of Multiplicity}

To compute the ratio of the number of systems of different degree of multiplicity among the stars studied, we use all the data that we gathered on the observations of these systems using different methods. Of the 109 stars considered 24 are spectral binaries (Goldberg et al., 2002 Latham et al., 2002); one star (G87-45) is a spectral triple (Latham et al., 2002); seven stars are speckleinterferometric binaries, and one star (G111-38) is a speckle-interferometric triple. Twelve stars have companions listed in the WDS catalog. It goes without saying that there are components detected using different methods. For example, the G102-20 binary with a period of 26 years (Latham et al., 2002) was found both spectroscopically and using speckle interferometry. Similarly, the outer pair in the triple system G111-38 $\left(\rho \approx 2^{\prime \prime}\right)$ can be detected both using speckle interferometry and visually.

The resulting ratio of the number of single, binary, triple, and quadruple systems discovered using all methods among the stars of our sample is equal to 71:28:6:1. The corresponding estimate for F7- to G9-type disk stars uncorrected for unresolved binaries (Duquennoy \& Mayor, 1991) is equal to 51:40:7:2. We point out an important difference between the two samples compared. Whereas our sample consists of stars selected by magnitude and spatial velocities, the sample used in Duquennoy \& Mayor, 1991 is only distance limited (all its stars are located within $22 \mathrm{pc}$ of the Sun).

\section{CONCLUSIONS}

We selected for observations with high angular resolution a total of 223 high proper motion metal-poor objects from the CLLA catalog (Carney et al., 1994). Our speckle-interferometric observations of 109 stars made with the 6-m telescope of SAO RAS allowed us to resolve eight stars into components and we were the first to astrometrically resolve seven objects. Additional data on spectral and astrometric multiplicity allowed us to estimate the ratio of the number of single, binary, triple, and quadruple systems to be 71:28:6:1.

In the next paper of this series we will continue to publish the results of our speckle-interferometric observations of the star sample presented.

Acknowledgements. This work makes use of the SIMBAD database and WDS catalog (Mason et al., 2001).

\section{References}

J. Norris, ApJS 61, 667 (1986).

S. R. Majewski, ARA\&A 31, 575 (1993).

H. A. Abt and S. G. Levi, AJ 74, 908 (1969).

H. A. Abt and D. W. Willmarth, Astrophys. J. 318, 786 (1987).

B. W. Carney and D. W. Latham, AJ 92, 116 (1987).

D. Goldberg, T. Mazeh, D. W. Latham, et al., AJ 124, 1132 (2002).

D. W. Latham, R. P. Stefanik, G. Torres, et al., AJ 124, 1144 (2002).

C. Allen, A. Poveda, and M. A. Herrera, A\&A 356, 529 (2000).

M. R. Zapatero Osorio and E .L. Martin, A\&A 419, 167 (2004).

H. Zinnecker, R. Kohler, and H. Jahreiss, Rev. Mex. Astrom. \& Astrophys. 21, 33 (2004).

B. W. Carney, D. W. Latham, J. B. Laird, et al., AJ 107, 2240 (1994) (CLLA).

H. L. Giclas, Jr. R. Burnham, and H. G. Thomas, Lowell Proper Motion Survey, Northern Hemisphere (Lowell Observatory, Flagstaff, 1971).

H. L. Giclas, Jr. R. Burnham, and H. G. Thomas, Lowell Obs. Bull. No 164 (1978).

M. I. Arifyanto, B. Fuchs, H. Jahreiss, R. Wielen, A\&A 433, 911 (2005).

M. A. C. Perryman, ESA, The Hipparcos and Tycho Catalogues (ESA Publ. Division, SP-1200, 1997).

A. F. Maksimov, Yu. Yu. Balega, U. Beckmann, et al., Bull. Spec. Astrofiz. Obs. 56, 102 (2003).

I. I. Balega, Y. Y. Balega, K.-H. Hofmann, et al., A\&A 385, 87 (2002).

SIMBAD database: http://simbad.u-strasbg.fr/simbad/sim-fid

D. W. Latham, T. Mazeh, B. W. Carney, et al., AJ 96, 567 (1988).

B. D. Mason, G. L. Wycoff, W. I. Hartkopf, et al., AJ 122, 3466 (2001).

A. Duquennoy and M. Mayor, A\&A 248, 485 (1991). 
Table 3. WDS components for stars of the sample

\begin{tabular}{|c|c|c|c|c|c|}
\hline Name & WDS companion & $\rho\left(^{\prime \prime}\right)$ & $\triangle m$ & Status & References \\
\hline G172-16 & $00386+4738$ OSO 7 & 8.4 & 5.85 & + & Zapatero Osorio \& Martin, 2004 \\
\hline G2-38 & 01270+1200LDS3282 & 24.6 & 5.5 & + & Allen et al., 2000 \\
\hline G172-61 & $01344+4844 \mathrm{ES} 2587$ & & & - & Zapatero Osorio \& Martin, 2004 \\
\hline G71-33 & 01452+0331LDS3306 & & & - & \\
\hline G74-5 & $02104+2948 B U P 29 A B$ & & & - & \\
\hline & $02104+2948 \mathrm{BUP} 29 \mathrm{AC}$ & & & - & \\
\hline & 02104+2948BUP 29AD & & & - & \\
\hline G37-26 & $03084+2620$ OSO 14 & & & - & Zapatero Osorio \& Martin, 2004 \\
\hline G246-38 & $03313+6644 \mathrm{OSO} 15$ & & & - & Zapatero Osorio \& Martin, 2004 \\
\hline G95-57A/G95-57B & $03470+4126 \mathrm{STF} 443 \mathrm{AB}$ & 7.4 & 0.62 & + & Allen et al., 2000 \\
\hline & $03470+4126 \mathrm{STF} 443 \mathrm{AC}$ & & & - & \\
\hline & $03470+4126 \mathrm{FOX} 135 \mathrm{CD}$ & & & - & Zapatero Osorio \& Martin, 2004 \\
\hline HD 25329 & $04033+3516$ OSO 16 & & & - & Zapatero Osorio \& Martin, 2004 \\
\hline G99-31W & 05449+0915HDS 769 & & & - & Perryman, 1997 \\
\hline $\mathrm{BD}+19^{\circ} 1185 \mathrm{~A}$ & 06032+1922HDS 823Aa & 0.2 & 2.12 & + & Perryman, 1997, * \\
\hline & 06032+1922LDS6195AB & 6.9 & 4.06 & + & Allen et al., 2000 \\
\hline G88-10 & 07104+2421OSO 19 & & & - & Zapatero Osorio \& Martin, 2004 \\
\hline G89-14 & $07224+0854 \mathrm{GIC} 72 \mathrm{AB}$ & & & - & Allen et al., 2000 \\
\hline & $07224+0854 \mathrm{ALC} 2 \mathrm{BC}$ & 34.0 & 6.3 & + & Allen et al., 2000 \\
\hline G112-43/G112-44 & 07437-0004HJ 2413 & 11.8 & 1.58 & + & Allen et al., 2000 \\
\hline G111-38 & $07495+4128 \mathrm{~A} 2468 \mathrm{AB}$ & 2.2 & 1.27 & + & Perryman, 1997, * \\
\hline & 07495+4128LDS 900AB-C & & & - & \\
\hline G90-25 & 07536+3036BUP 108 & & & - & Zapatero Osorio \& Martin, 2004 \\
\hline G251-54 & $08110+7955 \mathrm{LDS} 1668 \mathrm{AB}$ & 110.5 & 5.4 & + & Zapatero Osorio \& Martin, 2004 \\
\hline & 08110+7955PWS 3AC & & & $-?$ & \\
\hline & $08110+7955 \mathrm{OSO} 21 \mathrm{AD}$ & & & - & Zapatero Osorio \& Martin, 2004 \\
\hline G40-14 & 08161+1942LDS3781 & 98.0 & 7.6 & + & Allen et al., 2000 \\
\hline G113-22 & 08170+0001LDS3782 & & & - & \\
\hline G9-36 & $08580+2428 \mathrm{OSO} 23 \mathrm{AB}$ & & & - & Zapatero Osorio \& Martin, 2004 \\
\hline & $08580+2428 \mathrm{OSO} 23 \mathrm{AC}$ & & & - & Zapatero Osorio \& Martin, 2004 \\
\hline G115-49 & $09053+3848$ OSO 24 & & & - & Zapatero Osorio \& Martin, 2004 \\
\hline G120-15 & 11063+3113TDS7665AB & 7.6 & 0.68 & $+?$ & \\
\hline & $11063+3113 \mathrm{OSO} 36 \mathrm{AC}$ & & & - & Zapatero Osorio \& Martin, 2004 \\
\hline G10-4 & $11110+0625 \mathrm{OSO} 37$ & & & - & Zapatero Osorio \& Martin, 2004 \\
\hline G66-22 & $14433+0550$ OSO 58 & 3.2 & 3.19 & + & Zapatero Osorio \& Martin, 2004 * \\
\hline G23-14 & 19518+0537OSO 112 & & & - & Zapatero Osorio \& Martin, 2004 \\
\hline G143-33 & $20084+1503 \mathrm{OSO} 117 \mathrm{AB}$ & & & - & Zapatero Osorio \& Martin, 2004 \\
\hline & $20084+1503 \mathrm{OSO} 117 \mathrm{AC}$ & & & - & Zapatero Osorio \& Martin, 2004 \\
\hline & $20084+1503 \mathrm{OSO} 117 \mathrm{AD}$ & & & - & Zapatero Osorio \& Martin, 2004 \\
\hline & $20084+1503 \mathrm{OSO} 117 \mathrm{AE}$ & & & - & Zapatero Osorio \& Martin, 2004 \\
\hline & 20084+1503LDS1033AF & & & - & \\
\hline G125-64 & $20090+4252 \mathrm{OSO} 118 \mathrm{AB}$ & & & - & Zapatero Osorio \& Martin, 2004 \\
\hline & $20090+4252 \mathrm{OSO} 118 \mathrm{AC}$ & & & - & Zapatero Osorio \& Martin, 2004 \\
\hline
\end{tabular}


Table 4: Unresolved stars

\begin{tabular}{|c|c|c|}
\hline Name & Filter $(\lambda / \Delta \lambda, \mathrm{nm})$ & Epoch \\
\hline G172-16 & $800 / 100$ & 2006.9463 \\
\hline G33-30 & $800 / 100$ & 2006.9437 \\
\hline G2-38 & $800 / 100$ & 2006.9438 \\
\hline G172-58 & $800 / 100$ & 2006.9462 \\
\hline G172-61 & $550 / 20 ; 800 / 100$ & 2006.9462 \\
\hline G173-10 & $550 / 20 ; 800 / 100$ & 2006.9463 \\
\hline G2-50 & $800 / 100$ & 2006.9438 \\
\hline G71-33 & $800 / 100$ & 2006.9438 \\
\hline G245-32 & $550 / 20$ & 2006.9443 \\
\hline G133-45 & $800 / 100$ & 2006.9467 \\
\hline G71-55 & $800 / 100$ & 2006.9438 \\
\hline G72-60 & $800 / 100$ & 2006.9468 \\
\hline G74-5 & $550 / 20$ & 2006.9468 \\
\hline G74-30 & $800 / 100$ & 2006.9467 \\
\hline G36-47 & $800 / 100$ & 2006.9468 \\
\hline G37-26 & $550 / 20$ & 2006.9468 \\
\hline G5-19 & $550 / 20$ & 2006.9468 \\
\hline G221-7 & $550 / 20$ & 2006.9442 \\
\hline G5-35 & $800 / 100$ & 2006.9468 \\
\hline G246-38 & $800 / 100$ & 2006.9442 \\
\hline G79-42 & $800 / 100$ & 2006.9468 \\
\hline G79-43 & $800 / 100$ & 2006.9468 \\
\hline G79-56 & $800 / 100$ & 2006.9468 \\
\hline G95-57A & $550 / 20$ & 2006.9414 \\
\hline G95-57B & $550 / 20$ & 2006.9414 \\
\hline HD 25329 & $550 / 20$ & 2006.9414 \\
\hline G82-18 & $800 / 100$ & 2006.9415 \\
\hline G84-29 & $550 / 20$ & 2006.9415 \\
\hline G191-23 & $800 / 100$ & 2006.9448 \\
\hline G86-39 & $800 / 100$ & 2006.9415 \\
\hline G86-40 & $550 / 20 ; 800 / 100$ & 2006.9416 \\
\hline G96-48 & $800 / 100$ & 2006.9417 \\
\hline G99-31W & $550 / 20 ; 800 / 100$ & 2006.9417 \\
\hline G102-27 & $550 / 20$ & 2006.9471 \\
\hline G99-48 & $800 / 100$ & 2006.9471 \\
\hline G101-34 & $800 / 100$ & 2006.9471 \\
\hline G192-28 & $800 / 100$ & 2006.9448 \\
\hline G105-50 & $800 / 100$ & 2006.9471 \\
\hline G192-43 & $800 / 100$ & 2006.9448 \\
\hline G87-13 & $800 / 100$ & 2006.9417 \\
\hline G108-48 & $800 / 100$ & 2006.9445 \\
\hline G107-50 & $800 / 100$ & 2006.9475 \\
\hline G87-27 & $550 / 20 ; 800 / 100$ & 2006.9472 \\
\hline G88-10 & $800 / 100$ & 2006.9472 \\
\hline G108-58 & $800 / 100$ & 2006.9445 \\
\hline G88-27 & $800 / 100$ & 2006.9472 \\
\hline G90-3 & $800 / 100$ & 2006.9472 \\
\hline G88-32 & $800 / 100$ & 2006.9418 \\
\hline BD-1 ${ }^{\circ} 1792$ & $800 / 100$ & 2006.9446 \\
\hline G112-43 & $800 / 100$ & 2006.9446 \\
\hline G112-44 & $800 / 100$ & 2006.9446 \\
\hline G90-25 & $550 / 20 ; 800 / 100$ & 2006.9473 \\
\hline G251-54 & $800 / 100$ & 2006.9475 \\
\hline G234-24 & $800 / 100$ & 2006.9475 \\
\hline G40-8 & $800 / 100$ & 2006.9446 \\
\hline
\end{tabular}


Table 4: (Continued)

\begin{tabular}{l|c|c}
\hline \multicolumn{1}{c|}{ Name } & Filter $(\lambda / \Delta \lambda, \mathrm{nm})$ & Epoch \\
\hline G234-28 & $800 / 100$ & 2006.9448 \\
G40-14 & $800 / 100$ & 2006.9446 \\
G113-22 & $800 / 100$ & 2006.9446 \\
G194-22 & $800 / 100$ & 2006.9448 \\
BD+25 1981 & $550 / 20 ; 800 / 100$ & 2006.9473 \\
G46-5 & $800 / 100$ & 2006.9474 \\
G115-34 & $800 / 100$ & 2006.9473 \\
G9-36 & $800 / 100$ & 2006.9473 \\
G114-26 & $550 / 20$ & 2006.942 \\
G115-49 & $800 / 100$ & 2006.9473 \\
G46-31 & $800 / 100$ & 2006.9476 \\
G41-41 & $800 / 100$ & 2006.9476 \\
G195-34 & $800 / 100$ & 2006.9422 \\
G48-29 & $800 / 100$ & 2006.9476 \\
G116-45 & $800 / 100$ & 2006.9473 \\
G161-73 & $800 / 100$ & 2006.9476 \\
G43-3 & $545 / 30$ & 2006.2759 \\
G53-41 & $800 / 100$ & 2006.9421 \\
G44-30 & $800 / 100$ & 2006.9422 \\
G58-23 & $800 / 100$ & 2006.9422 \\
G196-48 & $800 / 100$ & 2006.9422 \\
G58-25 & $550 / 20$ & 2006.9422 \\
G146-76 & $800 / 100$ & 2006.9477 \\
G253-41 & $550 / 20 ; 800 / 100$ & 2006.9422 \\
G120-15 & $800 / 100$ & 2006.9449 \\
G10-4 & $800 / 100$ & 2006.9449 \\
BD+36 2165 & $800 / 100$ & 2006.9449 \\
HD 97916* & $550 / 20$ & 2006.945 \\
G56-30 & $800 / 100$ & 2006.9449 \\
G254-24 & $800 / 100$ & 2006.9423 \\
G147-62 & $800 / 100$ & 2006.945 \\
G121-12 & $800 / 100$ & 2006.9477 \\
G176-53 & $800 / 100$ & 2006.9423 \\
G122-51 & $550 / 20$ & 2006.945 \\
G66-22 & $545 / 30 ; 800 / 110$ & 2006.3747 \\
G166-45 & $545 / 30$ & 2006.3747 \\
G16-13 & $545 / 30$ & 2006.3748 \\
G16-20 & $545 / 30$ & 2006.3749 \\
G153-21 & $545 / 30$ & 2006.3749 \\
G170-47 & $545 / 30$ & 2006.4488 \\
G23-14 & $800 / 110$ & 2006.4517 \\
G23-20 & $800 / 110$ & 2006.4517 \\
G24-3 & $800 / 110$ & 2006.4518 \\
\hline & $800 / 110$ & 2006.4518 \\
G154 & $800 / 1100$ & 2006.4518 \\
\hline
\end{tabular}

